\title{
L'OCTOMITIASE \\ DES TRUITES ARC-EN-CIEL ET FARIO
}

\author{
par le Docteur BELLET,
}

Vétérinaire

L'octomitiase est une maladie parasitaire, contagieuse, atteignant les Truites d'élevage arc-en-ciel et fario.

Cette affection sévit à l'heure actuelle dans toute la France, et cause dans de très nombreuses salmonicultures, des dégâts considérables, particulièrement pendant la période allant du mois de Mai au mois d'Août.

La plupart du temps, l'octomitiase n'est du reste pas diagnostiquée par les pisciculteurs qui attribuent alors la mortalité anormale subie par leurs alevins à diverses causes, sans soupçonner l'existence de cette parasitose, aussi grave que banale.

\section{ÉTIOLOGIE}

L'agent causal est un protozoaire flagellé, appelé : "Octomitus salmonis ». Il est apporté par l'eau et infeste le poisson par voie digestive.

Sous certaines conditions favorables, il se développe jusqu'à pullulation dans la lumière de la première partie de l'intestin.

Description du parasite : Ce protozoaire a une taille d'environ $10 \mathrm{mu}$. sur $4 \mathrm{mu}$. Il affecte une forme d'ogive, l'extrémité pointue étant à l'arrière. Il est translucide et porte huit flagelles (d'où son nom) très fines et très longues. Les trois premières paires sont fixées sur la partie antérieure du corps et la dernière paire prolonge à l'arrière la partie postérieure.

Ce parasite existerait aussi sous une forme intra-cellulaire, difficile à observer (dans l'épaisseur de la muqueuse intestinale).

Mode d'examen : L'octomitus peut être examiné au microscope, par une technique simple et sans coloration à la portée de tout pisciculteur disposant de cet appareil. 
Lorsqu'il y a suspicion d'octomitiase dans un bassin, on prélève quelques alevins encore vivants à la grille d'aval.

Aprés ouverture de la cavité abdominale, on étire l'intestin à l'aide d'une petite pince et l'on en sectionne un segment. Ce segment devra être prélevé immédiatement en arrière des appendices pyloriques. On le pose alors sur une lame de verre. Tandis que la partie postérieure est immobilisée on le presse à l'aide d'une lame de bistouri, d'arrière en avant pour faire sortir le mucus par la partie antérieure. Ce mucus est ensuite étalé sur la lame et recouvert d'une lamelle.

S'il s'agit de tous petits alevins, l'opération est rendue délicate et on peut alors se contenter d'examiner le tube digestif lui-même, à condition de bien l'étaler entre lame et lamelle pour voir les parasites à travers les parois intestinales extrêmement minces. (A noter dans ce cas que la mise au point est assez difficile).

Le prélèvement examiné ne doit pas être trop sec, sans quoi les octomitus ne pourraient remuer, ni trop liquide, ce qui rendrait l'examen défectueux. L'étalement est alors examiné, d'abord au petit grossissement (exemple : oculaire $\times 6$ objectif $\times 10$ ).

Un œil exercé voit alors très nettement de petites images se déplaçant très vite dans le champ visuel.

On met ensuite en place un grossissement plus fort $(x 20)$. Les parasites apparaissent alors très nettement et principalement dans la périphérie de la préparation.

Ils sont reconnaissables à leur forme et surtout à leur mode de déplacement. En effet ils sont en mouvement perpétuel. allant et venant sans cesse, contournant les obstacles, et furetant partout.

Les flagelles sont difficilement visibles. Il faut pour les voir, un bon microscope, une luminosité de choix sans réfringence. Ce sont les deux flagelles terminaux qui se distinguent le plus facilement. Ils sont très longs, très flexibles, et animés de mouvements sinusoïdaux.

Pour voir les autres flagelles, il faut absolument immobiliser un octomitus par compression sur la lamelle. Le parasite tourne alors lentement sur place, et l'on peut voir tous ses flagelles ressemblant aux pattes d'une araignée.

L'existence d'octomitus sous forme libre en nombre plus ou moins grand, permet de porter à coup sûr, le diagnostic d'octomitiase.

Naturellement il est nécessaire de faire plusieurs examens pour se rendre compte de la gravité de l'atteinte.

Dans certains cas il y a une véritable pullulation de parasites qui sont aussi nombreux que des fourmis dans une fourmilière. division.

Mode de reproduction. - L'octomitus se reproduit par simple

On peut d'ailleurs remarquer au microscope des parasites anormalement allongés et étranglés en leur milieu, prêts à se diviser.

Cette multiplication est extrêmement rapide. 
Il suffit de quelques jours pour qu'un bassin entier d'alevins soit entièrement et sévèrement infesté.

Il existerait une forme kystique pouvant être excrétée avec les fécès et résistant longtemps dans l'eau. C'est cette forme, qui ingérée par le poisson, l'infesterait et redonnerait à nouveau, la forme mobile.

\section{SYMPTOMATOLOGIE}

10 Signes extérieurs. - L'octomitiase est une maladie à symptômes très discrets et se manifestant sous diverses formes - ce qui explique qu'elle est souvent ignorée du pisciculteur, ce qui la rend encore plus dangereuse.

Les petits alevins infestés (d'un poids individuel de $0 \mathrm{~g} 50$ à $1 \mathrm{~g}$ environ) deviennent maigres, apathiques et marquent peut d'appétit. Ils prennent une coloration foncée, et forment en queue de bassin des colonies de plus en plus importantes avant de venir mourir aux grilles.

Les alevins un peu plus forts, peuvent avec les mêmes symptòmes, présenter quelques mouvements rapides et désordonnés inhabituels. Les poissons vrillent un peu comme dans la costiase, mais généralement ce vrillage, au lieu de se produire contre le fond du bassin, a lieu en pleine eau.

Au stade Truitelles (de 6 à $10 \mathrm{~cm}$ ), les poissons peuvent manifester des signes de vrillage intense, comme dans le tournis véritable. Les sujets atteints se mettent brusquement à tourner en huit sur place et à toute vitesse, puis disparaissent dans le lot.

Les premiers jours on n'aperçoit que quelques poissons manifestant ces signes, puis rapidement leur nombre augmente et des quantités de Truitelles font la vrille dans toute la longueur du bassin.

Quant aux Truites d'un an et plus qui sont atteintes d'octomitiase, elles ne présentent souvent aucun signe particulier, sinon qu'elles deviennent maigres, apathiques, se groupant en queue de bassin.

On voit donc que tous ces symptômes n'ont rien de spécifique et peuvent aisément prêter a confusion.

$2^{\circ}$ Signes nécropsiques. - L'examen nécropsique simple ne peut pas permettre de poser à coup sûr le diagnostic d'octomitiase, pas plus que l'examen clinique.

Cependant, l'autopsie montre que toujours, les intestins des poissons parasités ont des parois particulièrement minces, translucides, fragiles, et contenant une sérosité tirant sur le jaune. Il y a donc entérite, mais ici il ne s'agit pas d'une entérite alimentaire mais d'une entérite parasitaire.

En définitive, l'examen microscopique est donc indispensable. 


\section{PATHOGENIE}

Conditions favorables au développement de l'octomitiase. L'octomitiase peut se développer sous l'influence de certaines conditions favorables, qu'il est utile de signaler aux pisciculteurs.

$1^{\circ}$ Surpopulation. - La surpopulation en alevins est un des grands facteurs responsables du développement de la maladie. Les alevins restant en particulier longtemps en " laboratoire ", parqués en grand nombre dans de petites cuves, risquent presque à coup sûr de devenir les victimes de l'octomitus.

La contagion est alors extrêmement rapide, ce qui est naturel dans une telle promiscuité.

$2^{\circ}$ Qualité des eaux. - Certaines eaux sont particulièrement favorables à son développement et sans que l'on sache pourquoi. Les eaux de source les plus limpides n'échappent pas à cette règle. On dirait même qu'elles sont un facteur favorisant pour des alevins qui y stationnent trop longtemps - sans doute par insuffisance de qualités nutritives, ou encore par taux d'oxygène trop faible.

Une pisciculture qui a été victime d'une crise d'octomitiase une année, doit donc les années suivantes ètre particulièrement surveillée du point de vue de cette parasitose.

Dans certaines d'entre elles l'octomitus sévit à l'état endémique, principalement dan les piscicultures anciennes.

$3^{\circ}$ Troubles nutritionnels. - L'octomitus est un parasite, sa pathogénie suit donc la règle générale propre aux parasitoses.

Tout trouble de la nutrition, " en hypo " ou " en hyper ", favorise le développement, voire la pullulation de cet hôte intestinal.

C'est ainsi que des Truitelles d'un an provenant de a queues de production ", sous alimentées pendant la période hivernale, peuvent, nous l'avons constaté, être décimées par une octomitiase chronique et sévère.

A l'opposé, de jeunes alevins suralimentés, soit par une ration trop importante en poids, soit par une ration hyper-protidique (alevins nourris trop longtemps à la rate), sont souvent victimes de l'octomitiase.

Fait particulièrement important et sur lequel nous aurons l'occasion de revenir, certaines maladies, dites " à gros yeux", des alevins arc-en-ciel sont accompagnées la plupart du temps d'une octomitiase intense.

40 Maladies infectieuses et tous phénomènes amoindrissant la résistance des poissons. - L'octomitiase peut aussi trouver un terrain favorable, sur des Truites ayant subi diverses maladies infectieuses.

C'est ainsi qu'elle est assez fréquente après la furonculose des alevins ou après les entérites infectieuses du genre salmonellose.

En général, tout facteur diminuant la résistance du poisson peut favoriser le développement de.cette parasitose. 
Nous citerons, en particulier, l'emploi abusif de sulfamides ou d'antibiotiques, ou encore la transplantation d'alevins dans des eaux par trop différentes de leurs eaux d'origine.

Telles sont donc passées en revue, les principales conditions rendant plus aisée l'apparition de cette maladie.

Est-ce à dire que l'octomitiase, n'est et ne peut être qu'une maladie secondaire, comme " la mousse $n$, n'apparaissant que sur des sujets amoindris ?

C'est là une opinion assez répandue et de là à considérer cette parasitose comme une affaire bénigne, il n'y a qu'un pas, souvent allégrement franchi.

Or, il s'agit là d'une erreur manifeste.

L'octomitiase est une maladie grave, pouvant apparaitre même dans des cheptels irréprochables, et y causer très vite de lourds dégâts.

\section{Différentes formes de la maladie.}

La maladie peut évidemment sévir sous différentes formes, ceci en fonction de l'importance de l'infestation, et des diverses contingences ambiantes.

On peut distinguer :

10 Une forme aiguë, amenant une mortalité énorme et pouvant anéantir un cheptel de 4 à 500.000 alevins et plus en deux à trois semaines ;

$2^{\circ}$ Des formes subaiguës atteignant les différents bassins les uns après les autres, avec une allure plus lente;

$3^{\circ}$ Une forme chronique particulière aux Truites de 10 à 12 mois et sévissant en hiver;

$4^{\circ}$ Des formes à récidives.

Sous un autre angle, il existe des octomitiases pures et des octomitiases accompagnées par d'autres parasitoses (costias, gyrodactyles, vers intestinaux et surtout très fréquemment champignons intestinaux du genre saprolégnés, etc.), ou encore des octomitiases allant de pair avec des troubles de la nutrition (c'est le cas le plus fréquent) ou avec des maladies infectieuses, ce qui rend le diagnostic étiologique malaisé.

\section{DIAGNOSTIG}

Cliniquement, il est difficile de poser le diagnostic d'octomitiase. On ne peut établir ainsi qu'une suspicion.

Celle-ci doit être confirmée par l'examen microscopique, le seul permettant d'affirmer à coup sûr l'existence d'octomitiase.

A ce sujet nous précisons que les parasites existant dans les étalements préparés sur lame de verre, meurent au bout de quelques minutes 
et deviennent de ce fait pratiquement impossible à déceler. Ils se recroquevillent, s'immobilisent, et comme ils sont translucides, ils ne peuvent plus être distingués par examen direct.

D'autre part, les parasites peuvent continuer à vivre un certain temps après la mort du poisson mais à condition que celui ne se décompose pas.

Par conséquent, le diagnostic microscopique d'octomitiase, le meilleur et le plus sûr, est celui pratiqué sur place, à la pisciculture.

Ce diagnostic peut cependant être porté à partir de sujets pris vivants et expédiés dans un laboratoire compétent, glacés et placés sous caisse isothermique.

Mais dans ce cas, il est indispensable que l'emballage soit fait très correctement et que le voyage des prélèvements n'excède pas vingtquatre heures.

\section{DIAGNOSTIG DIFFERENTIEL}

Du point de vue clinique, signalons que l'octomitiase est la plupart du temps confondue par les pisciculteurs avec la costiase ou avec l'entérite banale, quant elle n'est pas totalement ignorée.

Comme elle accompagne fréquemment, diverses maladies dites * des gros yeux , et qu'elle les aggrave sérieusement, elle doit être alors détectée précocément.

Du point de vue microscopique il n'y a pas de confusion possible.

\section{PRONOSTIC}

L'octomitiase est une maladie grave, capable à elle seule de décimer totalement en quelques semaines un cheptel important.

Elle est d'autant plus grave qu'elle est à l'heure actuelle en France très sous-estimée, comme nous avons pu nous en rendre compte.

Elle est d'autant plus dangereuse que sa symptomatologie est polymorphe et discrète.

Sa gravité est augmentée par les erreurs alimentaires si fréquentes en pisciculture. C'est ainsi qu'en association avec une alimentation excessive en protides l'octomitiase jointe au syndrôme anémie et exophtalmie des alevins, est responsable de Mai à Août dans notre pays de $\mathbf{8 0} \%$ environ de la mortalité totale des alevins.

Nous pouvons donc affirmer que l'octomitiase détruit à chaque saison d'alevinage des millions d'élèves et qu'elle sévit dans toutes les régions de France sans exception.

Elle est la maladie parasitaire type de l'élevage industriel des Truites. Le pisciculteur doit la considérer comme un des principaux ennemis de ses alevins.

Elle est beaucoup plus dangereuse que la costiase, qui elle, est la seule maladie contre laquelle les pisciculteurs appliquent une prophylaxie rationnelle. 
En résumé, on peut dire pour trouver un point de comparaison, que l'octomitiase est à la salmoniculture, ce qu'est la coccidiose à l'aviculture.

\section{TRAITEMENT}

10 Traitement prophylactique. - Il consiste surtout à éviter les causes favorisantes, telles celles indiquées antérieurement, et tout spécialement la surpopulation.

$2^{\circ}$ Traitement curatif. - Plusieurs médicaments sont à notre disposition pour détruire ce parasite intestinal.

a) Le stovarsol. - C'est un dérivé arsenical très bon marché. Il a été recommandé par quelques auteurs.

Nous pouvons affirmer que c'est là un piètre médicament contre l'octomitiase et nous ne le conseillons pas.

b) Le carbarsone. - C'est un autre dérivé de l'arsenic. Il est chaudement recommandé par les Américains.

Nous avons pu constater qu'il est très bien supporté par les alevins et qu'il est efficace. Mais son prix de revient élevé et la difficulté que l'on a à s'en procurer en quantité importante, sont cependant deux inconvénients majeurs. Commercialement il se présente sous la forme d'une poudre blanche, insoluble dans l'eau. La posologie à utiliser est de l'ordre de 2 grammes par kilo de nourriture, de 4 à 7 jours de suite.

c) Le Naphtol $B$. - Le naphtol $\mathrm{B}$ est aussi un bon médicament et peu onéreux.

Poudre blanche, légère, à saveur très amère, elle doit être utilisée à la dose de 5 grammes par kilo de nourriture pendant 4 à 5 jours consécutifs.

Les alevins ne supportent que passablement ce produit dont l'efficacité est pourtant certaine. Cependant son goût très prononcé amène souvent les poissons à refuser rapidement la nourriture dans laquelle ce produit est incorporé.

d) Le Calomel. - C'est du protochlorure de mercure.

Poudre blanche, très lourde, devant être conservée à l'abri de la lumière, elle a une action remarquable contre l'octomitus.

La dose à employer est de 2 grammes par kilo de nourriture pendant 4 jours consécutifs. Les poissons acceptent très bien ce médicament et le supportent de même sans diminution d'appétit.

Le gros avantage de ce produit est son action favorable sur le foie.

C'est en effet un cholagogue très anciennement utilisé tant en médecine vétérinaire qu'en médecine humaine.

Ajoutons à cette propriété, celle d'être un très bon antiseptique intestinal, et l'on comprendra que nous recommandons particulièrement le Calomel, comme traitement de l'octomitiase des alevins, si souvent accompagnée de stéatose hépatique. 
Tous ces produits doivent être incorporés à la nourriture.

Mais cette opération doit être faite minutieusement, surtout lorsqu'il s'agit du Calomel. En effet la densité de celui-ci, jointe à sa posologie réduite, font que le volume du médicament à utiliser est minime.

Il faut donc d'abord faire un pré-mélange de la drogue dans une farine quelconque, par exemple de la mouture d'orge, mélange rendu malaisé à cause de la différence de densité.

Ensuite cette première mixture devra être introduite dans la nourriture.

Si le pisciculteur posséde un mélangeur, l'opération est facile et le mélange sera homogène; mais s'il ne dispose pas de cette machine - pourtant si nécessaire - il devra se contenter de saupoudrer la pâtée avec le pré-mélange et de passer la nourriture plusieurs fois de suite au broyeur.

Ces précisions pourraient paraitre superflues, mais il ne faut pas omettre que dans un élevage de masse, l'action d'un médicament est autant fonction de son mode de distribution que de sa valeur intrinsèque.

D'une façon générale, ces traitements (carbarsone, naphtol $\mathrm{B}$, ou Calomel), provoquent pendant les premiers jours un accroissement très net de la mortalité.

Il n'y a pas lieu de s'en inquiéter. Ce sont tous les alevins affaiblis, particulièrement parasités, donc de toute façon condamnés, qui meurent de suite, sous l'action du traitement, et cela est bien ainsi.

Au bout de cinq à six jours, la mortalité diminue nettement, l'aspect général des lots traités s'améliore, l'appétit revient ainsi que la vivacité (si l'octomitiase est la seule maladie en cause bien entendu).

Dans certains cas, il faudra cependant faire un nouveau traitement 15 jours ou trois semaines après le premier, en particulier dans les piscicultures très infestées.

Il arrive aussi lorsqu'on traite trop tardivement, qu'un certain nombre de sujets refusant toute nourriture, n'absorbent pas alors le médicament distribué, et restent de cette façon pendant leur survie, des porteurs de parasites. Ceux-ci peuvent à nouveau se multiplier et provoquer une rechute générale.

C'est pourquoi dans ce cas nous recommandons après le traitement au Calomel de faire un bain de permanganate à dose assez forte pour éliminer tous les alevins particulièrement affaiblis. Cet antiseptique combattra avec succès les complications de mousse et détruira les sujets dont la survie représente un danger pour le reste du cheptel.

Il va sans dire qu'après ces traitements les alevins devront recevoir des soins attentifs et, en particulier, un régime de choix, riche en vitamines, afin de leur permettre de retrouver rapidement une bonne santé sans suralimentation.

$3^{\circ}$ Traitement préventif. - Il n'y a pas à proprement parler de traitement préventif de l'octomitiase.

Cependant nous considérons et nous en sommes persuadés, que 
bien des déboires seraient évités si les pisciculteurs traitaient systématiquement leurs alevins, par une cure de Calomel, ce qui n'est ni difficile, ni dangereux, ni coûteux, une à deux fois de Mai à Août, la date de ces traitements étant fonction de l'âge des alevins ainsi que des contingences propres aux eaux de l'établissement.

Il serait également bon de traiter en automne ou en hiver les lots retardataires de Truitelles, la plupart du temps porteurs d'octomitus, et qui risquent au printemps suivant d'être les vecteurs de contagion.

La lutte contre les parasites externes (costias, saprolégnés, etc.), est entrée dans les mœurs des salmoniculteurs. Le bénéfique formol et le apricieux sulfate de cuivre sont en effet largement utilisés.

La lutte contre les parasites internes, doit à son tour être entreprise, et ceci d'une façon systématique.

L'octomitus est l'un de ces parasites internes, actuellement le plus répandu et le plus nocif.

Nous tenions à attirer l'attention des salmoniculteurs sur les dangers qu'il représente sur les lourdes pertes qu'il fait subir à leurs alevins. et donc sur l'intérêt qu'ils ont à le combattre efficacement en temps voulu, 